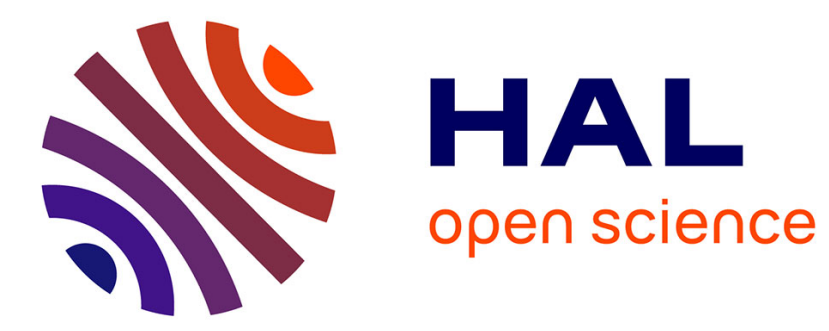

\title{
Magnetic Investigation of an Atypical Three-Dimensional Bimetallic Oxalate-Based Magnet
}

Virginie Simonet, Françoise Damay, Sylvie Ferlay, Andrew Cea, Catalin Maxim, Cyrille Train

\section{To cite this version:}

Virginie Simonet, Françoise Damay, Sylvie Ferlay, Andrew Cea, Catalin Maxim, et al.. Magnetic Investigation of an Atypical Three-Dimensional Bimetallic Oxalate-Based Magnet. Journal of Physical Chemistry C, 2020, 124 (1), pp.952-957. 10.1021/acs.jpcc.9b10599 . hal-02438582

\section{HAL Id: hal-02438582 https://hal.science/hal-02438582}

Submitted on 18 Nov 2020

HAL is a multi-disciplinary open access archive for the deposit and dissemination of scientific research documents, whether they are published or not. The documents may come from teaching and research institutions in France or abroad, or from public or private research centers.
L'archive ouverte pluridisciplinaire HAL, est destinée au dépôt et à la diffusion de documents scientifiques de niveau recherche, publiés ou non, émanant des établissements d'enseignement et de recherche français ou étrangers, des laboratoires publics ou privés. 


\title{
Magnetic Investigation of an Atypical Three-Dimensional Bimetallic Oxalate-Based Magnet
}

\author{
Virginie Simonet, ${ }^{\dagger}$ Françoise Damay, ${ }^{\S}$ Sylvie Ferlay, ${ }^{\| \odot}$ Andrew Cea, ${ }^{\dagger} \perp$ Catalin Maxim, ${ }^{\ddagger}, \#$ \\ and Cyrille Train**; \\ ${ }^{\dagger}$ Institut Néel, CNRS and Université Grenoble Alpes, B.P. 166, 38042 Grenoble Cedex 9, France \\ ${ }^{\ddagger}$ LNCMI, CNRS, EMFL, Université Grenoble Alpes, INSA Toulouse, Université Toulouse Paul Sabatier, 38000 Grenoble, France \\ ${ }^{\S}$ Laboratoire Léon Brillouin, CEA-CNRS, CEA/Saclay, F-91191 Gif sur Yvette, France \\ "University of Strasbourg, UMR Unistra-CNRS 7140, Institut le Bel, 4 rue Blaise Pascal, F-67000 Strasbourg, France
}

ABSTRACT: Detailed magnetic measurements and powder neutron diffraction studies have been performed on a three-dimensional (3D) oxalate-based metal-organic framework $\left(\mathrm{NH}_{4}\right)_{5}\left[\mathrm{Mn}_{2} \mathrm{Cr}_{3}(\text { ox })_{9}\right] \cdot 10 \mathrm{H}_{2} \mathrm{O}$ $\left(\mathrm{ox}^{2-}=\mathrm{C}_{2} \mathrm{O}_{4}{ }^{2-}\right)$. The thermal variations of the magnetic susceptibilitiy measured via field-cooled and zero-field-cooled procedures are compatible with an antiferromagnetic spin organization in the presence of some single-ion anisotropy. This is supported by the metamagnetic behavior observed at $0.7 \mathrm{~T}$ in the magnetization curve at $2 \mathrm{~K}$. Temperature-dependent neutron diffraction experiments have been performed between 2.5 and $300 \mathrm{~K}$ on the powdered sample. The structural part of the neutron diffraction pattern matches with the $\mathrm{C} 2 / \mathrm{c}$ space group found by single-crystal X-ray diffraction. Below $6 \mathrm{~K}$, the
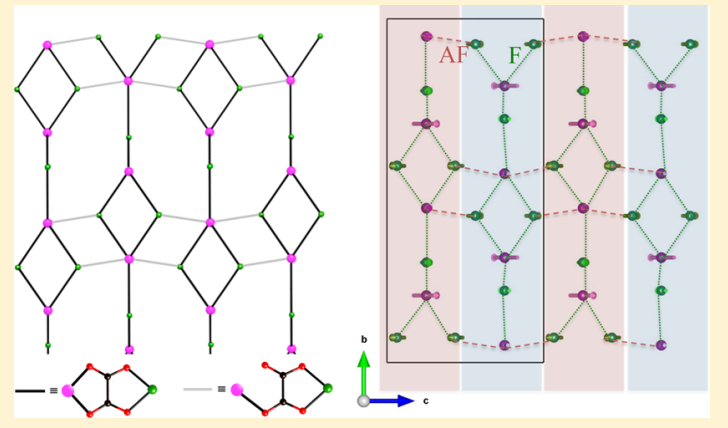
long-range magnetic ordering leads to the observation of a single magnetic peak at $0.386 \AA^{-1}$. The refinement of the neutron diffractogram allows to propose a magnetic structure where the magnetic moments of the manganese(II) and chromium(III) ions are noncollinear with a maximum angle of almost $70^{\circ}$. In agreement with an orbital analysis, the coupling between these magnetic moments bridged in a bis(bidentate) oxalate ligand is mainly ferromagnetic whereas it is mainly antiferromagnetic for metal ions bridged in a monodentate-bidentate mode.

\section{INTRODUCTION}

Along the years, oxalate-based networks have been demonstrated to be a versatile platform toward multifunctional molecular magnets. ${ }^{1}$ This is related to the ability of the negatively charged bimetallic oxalate-based networks to welcome a wide variety of (functional) cations and to the possibility to synthesize oxalate-based compounds in optically active forms. $^{2}$

Recently, in particular, to develop protonic conduction in these materials, ${ }^{3,4}$ cations enabling hydrogen bonds were introduced in oxalate-based magnets. ${ }^{3-7}$ These weak intermolecular interactions, that compete with the coordination bonds of the bimetallic oxalate network, have deeply enlarged the structural landscape of these materials, which was dominated by the two-dimensional (2D) $(6,3)$ and threedimensional (3D) $(10,3)$ coordination networks discovered in the 90s. ${ }^{2,8,9}$ Such H-bond active cations have sometimes limited the dimensionality of the formed coordination architectures $^{10,11}$ or induced atypical $2 \mathrm{D}$ and $3 \mathrm{D}$ structures. $4,6,7,12,13$ In these latter compounds, the metallic ions are indeed coordinated from 2 to 5 bridging oxalate ligands. This is in line with the numerous coordination modes of the oxalate anion: nonbridging, monodentate-bidentate as well as bis(bidentate) bridging modes. ${ }^{14}$ This structural versatility strongly influences the magnetic exchange interactions between the metallic ions toward original magnetic behaviors. $^{6,12}$

In this contribution, this specificity brought by the use of different template cations is further demonstrated by the thorough exploration of the magnetic behavior of $\left(\mathrm{NH}_{4}\right)_{5}\left[\mathrm{Mn}_{2} \mathrm{Cr}_{3}(\mathrm{ox})_{9}\right] \cdot 10 \mathrm{H}_{2} \mathrm{O}:^{6}$ it is investigated by detailed macroscopic magnetization measurements and its magnetic structure is determined by neutron diffraction.

\section{EXPERIMENTAL SECTION}

General Details. $\left(\mathrm{NH}_{4}\right)_{5}\left[\mathrm{Mn}_{2} \mathrm{Cr}_{3}(\mathrm{ox})_{9}\right] \cdot 10 \mathrm{H}_{2} \mathrm{O}$ polycrystalline sample has been synthesized according to the reported procedure.

The IR spectra were recorded on a Nicolet iS50 FT-IR spectrometer as $\mathrm{KBr}$ pellets in the $4000-250 \mathrm{~cm}^{-1}$ region. The powder X-ray diffraction patterns were collected at room temperature on a Philips PW-1050 goniometer using the Bragg-Brentano configuration and Mn-filtered Fe $\mathrm{K} \alpha$ radiation. The quality of the samples was checked by IR

Received: November 12, 2019

Revised: December 9, 2019

Published: December 10, 2019 
(a)

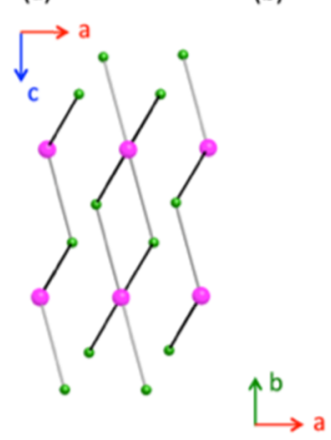

(b)

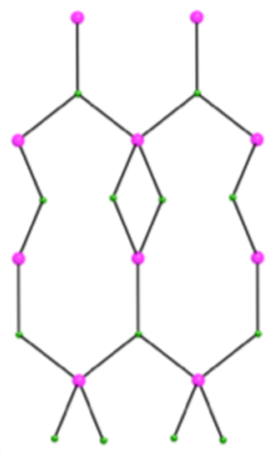

(c)

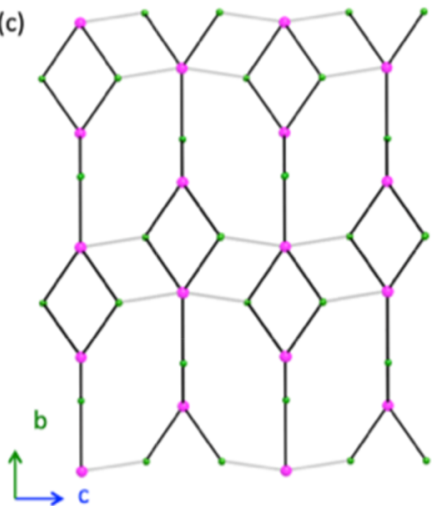

Figure 1. Projection of the bimetallic network in the (a) $a c$ plane, (b) $a b$ plane, and (c) $b c$ plane. The manganese and chromium ions are represented in magenta and green, respectively. The black and gray lines represent the oxalate bridges in a bis(bidentate) and monodentatebidentate bridging modes, respectively (adapted from ref $^{6}$ ).

spectroscopy and powder X-ray diffraction, comparing the experimental data with the published ones. ${ }^{6}$

Magnetic Studies. Magnetization measurements versus temperature $(2-300 \mathrm{~K})$ were performed on powdered samples using a SQUID magnetometer (Quantum Design MPMS-XL), applying magnetic fields between $10^{-3}$ and $10^{-1} \mathrm{~T}$. Both fieldcooled (FC) and zero-field-cooled (ZFC) procedures were used, with the measurements being performed while increasing the temperature after cooling with or without magnetic field, respectively. Isothermal magnetization versus field up to $7 \mathrm{~T}$ was recorded at $2 \mathrm{~K}$. All data were corrected for the contribution of the sample holder and the diamagnetism of the samples estimated from Pascal's constants. ${ }^{15}$

Neutron Diffraction. The neutron diffraction experiments were performed at the Laboratoire Léon Brillouin (CEA Saclay) using the G4.1 powder neutron diffractometer equipped with a multidetector of 800 cells. The powder sample was set in a cylindrical vanadium container and cooled down in a cryostat. Diffraction patterns were recorded at $\lambda=$ $2.426 \AA$ in the $6-85.9^{\circ} 2 \theta$ range, at different temperatures between 2.5 and $300 \mathrm{~K}$. The analysis of the diffraction patterns was performed at different temperatures using the FullProf software. ${ }^{16}$ The lattice parameters were refined to match the exact experimental peak positions. Combined with a symmetry analysis performed using the Basireps software of the FullProf Suite, this enables to obtain a model for the magnetic structure of the compound below $T_{\mathrm{N}}$.

\section{RESULTS AND DISCUSSIONS}

The magnetic properties of $\left(\mathrm{NH}_{4}\right)_{5}\left[\mathrm{Mn}_{2} \mathrm{Cr}_{3}(\mathrm{ox})_{9}\right] \cdot 10 \mathrm{H}_{2} \mathrm{O}$ have been studied starting from the preliminarily published investigations, where a magnetic ordering temperature $T_{\mathrm{N}}$ was observed at $6.0 \mathrm{~K}^{6}$ In our previous work, the magnetic susceptibility was found to be compatible with a global antiferromagnetic (AF) long-range magnetic order (LRMO) involving dominant ferromagnetic (F) interactions. This apparently puzzling situation was tentatively explained by a magnetostructural analysis: in the $(a b)$ planes, all the oxalate ligands are in a bis(bidentate) bridging mode (Figure 1b) which is believed, by comparison with the situation encountered in classical (2D) $(6,3)^{8}$ and three-dimensional (3D) $(10,3)^{17}$ networks, to promote $\mathrm{F}$ exchange interactions between the spin bearers, leading to the formation of ferromagnetically ordered planes. In the $(b c)$ planes (Figure 1c), along the $b$ axis, bis(bidentate) bridging oxalate ligands are retrieved (black sticks in Figure 1c) whereas along the $c$ axis, the oxalate ligands adopt a bidentate-monodentate mode (gray sticks in Figure 1c) which is believed, by comparison with a published case of a rather asymmetrical oxalate bridge, ${ }^{12}$ to convey AF exchange interaction. Accordingly, this interaction should induce an antiparallel orientation of the magnetization borne by adjacent $(a b)$ planes leading to an overall AF LRMO.

To confirm this assumption with robust experimental proofs, detailed macroscopic magnetometric studies are combined with neutron diffraction experiments allowing a microscopic insight into the LRMO.

Magnetometric Studies. The magnetization of $\left(\mathrm{NH}_{4}\right)_{5}\left[\mathrm{Mn}_{2} \mathrm{Cr}_{3}(\mathrm{ox})_{9}\right] \cdot 10 \mathrm{H}_{2} \mathrm{O}$ has been measured starting from $2 \mathrm{~K}$ up above the ordering temperature after cooling the sample with (FC) and without (ZFC) applying an external magnetic field. For both measuring modes, there is a clear-cut change in the thermal variations of the magnetization (Figure 2a): upon temperature increase, the FC (respectively ZFC)

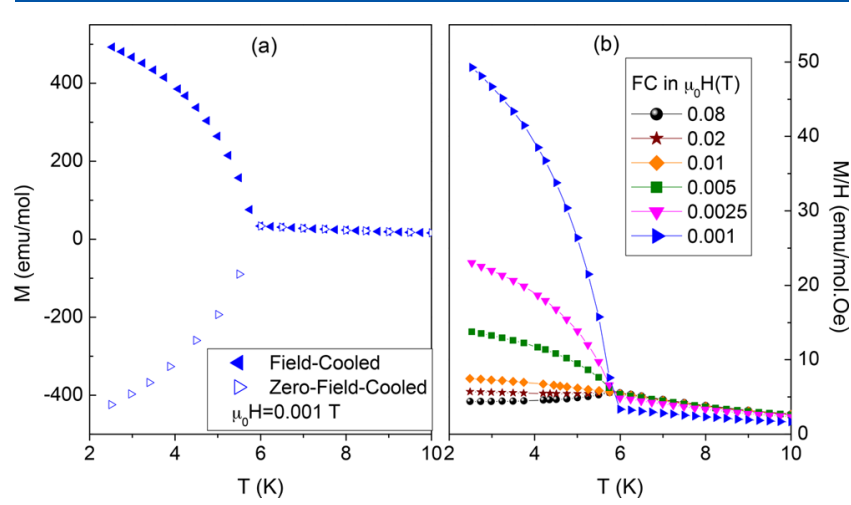

Figure 2. For $\left(\mathrm{NH}_{4}\right)_{5}\left[\mathrm{Mn}_{2} \mathrm{Cr}_{3}(\mathrm{ox})_{9}\right] \cdot 10 \mathrm{H}_{2} \mathrm{O}$ : (a) Magnetization versus temperature measured through a field-cooled (FC) procedure (filled symbols) and zero-field-cooled procedure (ZFC) (empty symbols) in a magnetic field of $10^{-3} \mathrm{~T}$. (b) Field-cooled measurements in various magnetic fields. The magnetic ordering temperature occurs just below $6 \mathrm{~K}$ when the curves depart from each other.

curve decreases (respectively increases) rapidly. At $6 \mathrm{~K}$, they merge and above this temperature, they decrease simultaneously at a much slower rate. This abrupt change in the thermal variations of the magnetization is a clear confirmation of the onset of an LRMO at $6 \mathrm{~K}$. The difference between the FC and ZFC measurements can be related to different 
processes: spin glass transition, selection of magnetic domains accompanying the onset of a ferromagnetic LRMO, contribution of the magnetic moments associated with magnetic domain walls or defects for an AF LRMO in the presence of some anisotropy. The latter can be either due to single-ion anisotropy or to anisotropic interaction. The FC curves were measured in fields varying between 0.001 and $0.08 \mathrm{~T}$ (Figure 2a). When increasing the field, the reduced magnetization $M$ / $\mathrm{H}$ below the ordering temperature is gradually overcome by the antiferromagnetic susceptibility, indicating that the compound is essentially an antiferromagnet.

The magnetization versus field in the ordered phase is shown in Figure 3. It saturates around $18 \mu_{\mathrm{B}}$ after a

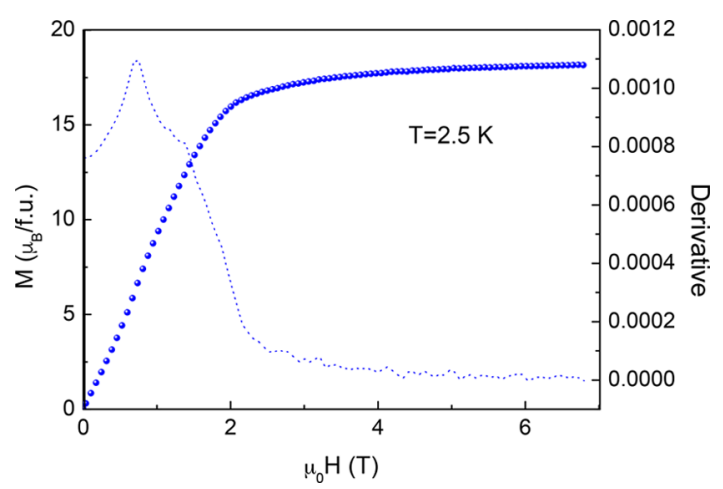

Figure 3. Magnetization of $\left(\mathrm{NH}_{4}\right)_{5}\left[\mathrm{Mn}_{2} \mathrm{Cr}_{3}(\mathrm{ox})_{9}\right] \cdot 10 \mathrm{H}_{2} \mathrm{O}$ versus field at $2.5 \mathrm{~K}$ (blue dots). The derivative is also shown (dotted line and right axis) evidencing a metamagnetic process from the maximum at $0.7 \mathrm{~T}$.

metamagnetic process occurring around $0.7 \mathrm{~T}$ best seen in the derivative of the magnetization (Figure 3). This indicates a spin reorientation under magnetic field, overcoming an anisotropy barrier, toward a ferromagnetic state compatible with a full saturation of $2 \mathrm{Mn}(\mathrm{II})\left(2 \times 5 \mu_{\mathrm{B}}\right.$ with $\left.g=2\right)$ and 3 $\mathrm{Cr}(\mathrm{III})\left(3 \times 3 \mu_{\mathrm{B}}\right.$ with $\left.g=2\right)$ per formula unit.

Altogether, the magnetization studies indicate the onset of an AF LRMO influenced by an extra phenomenon that can be single-ion zero-field splitting (ZFS) or anisotropy of the exchange interaction induced by antisymmetric exchange interaction. A distinction between these two possibilities can be provided by the microscopic vision brought about by neutron scattering experiments.
Neutron Diffraction Study. Powder neutron diffraction (PND) patterns of $\left(\mathrm{NH}_{4}\right)_{5}\left[\mathrm{Mn}_{2} \mathrm{Cr}_{3}(\mathrm{ox})_{9}\right] \cdot 10 \mathrm{H}_{2} \mathrm{O}$ have been recorded between 2.5 and $300 \mathrm{~K}$. The neutron diffraction patterns are composed of thin peaks, indicating a good crystallinity of the sample. Figure 4 shows the experimental pattern at $300 \mathrm{~K}$ together with a profile matching fit performed using the $C 2 / c$ space group observed by single-crystal $\mathrm{X}$-ray diffraction. ${ }^{6}$ All the peaks, except the one around $2 \theta=20.5^{\circ}$, are perfectly indexed, and the refined lattice parameters for the unit cell are: $a=8.845(1) \AA, b=35.454(4) \AA, c=16.265(2) \AA$, $\alpha=90^{\circ}, \beta=93.395(7)^{\circ}, \gamma=90^{\circ}$, and $V=5084(1) \AA^{3}$. Note that the additional peak is not indexed even in lower symmetry subgroups, indicating that it must be extrinsic. The structural parameters found by PND are in good agreement with those determined from single-crystal X-ray diffraction at $173 \mathrm{~K}(a=$ 8.8345(1) $\AA$, $b=35.591(4) \AA$, $c=16.246(2) \AA, \alpha=90^{\circ}, \beta=$ $93.437(6)^{\circ}, \gamma=90^{\circ}$, and $\left.V=5098.8(1) \AA^{3}\right)$.

The temperature evolution of the structural parameters was deduced from the profile matching performed using the diffractograms recorded while lowering the temperature (Figure 5). No structural phase transition is observed between room temperature and $2.5 \mathrm{~K}$. Down to the magnetic ordering temperature, a compression of the $a$ and $c$ by less than $1 \%$ and, to a lesser extent, $b(0.17 \%)$ lattice parameters is observed. The monoclinic angle $\beta$ first decreases by $0.3^{\circ}$ down to $200 \mathrm{~K}$ before increasing by $0.15^{\circ}$ down to $T_{\mathrm{N}}$. It should be noted that, given the good crystallinity of the sample, the high incoherent background due to the large number of hydrogens decreased the signal-to-noise ratio but did not prevent a precise determination of the lattice parameters. The indeterminate positions of the hydrogen atoms in the compound prevented however a complete Rietveld refinement of the atomic positions in the neutron diffractograms.

When lowering the temperature through the ordering temperature, no extra peaks appear on the neutron diffraction pattern. However, the evolution of the intensity is particularly visible for the peak at $2 \theta=8.6^{\circ}$ (Miller's indices (001)), as observed in the inset of Figure 4. The thermal studies show that the intensity of this peak increases below $6 \mathrm{~K}$ attesting a magnetic transition, in accordance with the temperature found by magnetometry (Figure 2). The increase of the peak intensity is related to the superposition of the magnetic contribution, induced by the long-range ordering of the magnetic moments of $\mathrm{Mn}$ (II) and $\mathrm{Cr}$ (III) ions, on top of the
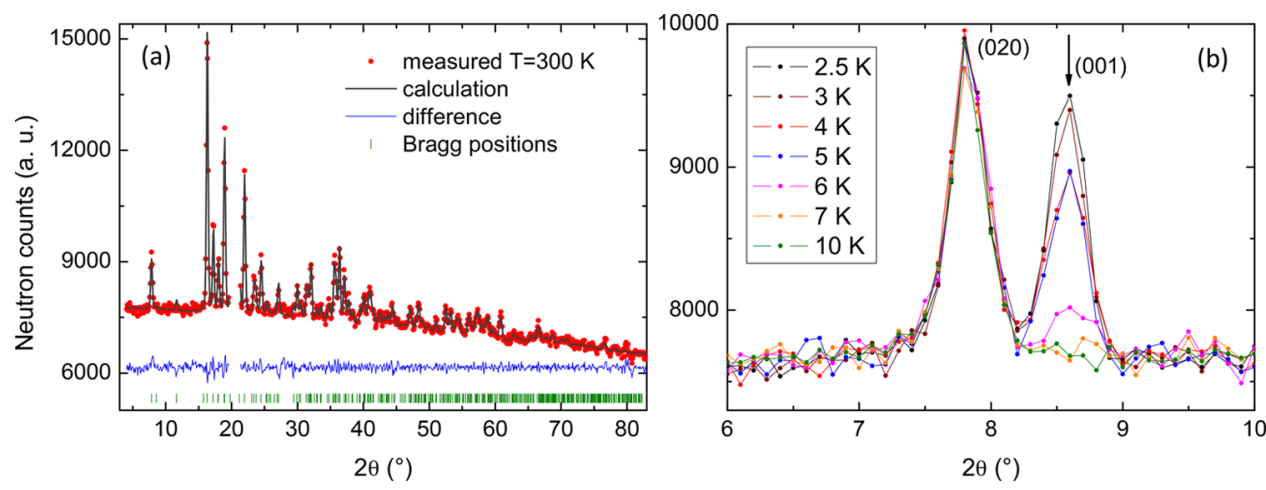

Figure 4. (a) Experimental neutron diffraction pattern (red circles) of a polycrystalline powder of $\left(\mathrm{NH}_{4}\right)_{5}\left[\mathrm{Mn}_{2} \mathrm{Cr}_{3}(\mathrm{ox})_{9}\right] \cdot 10 \mathrm{H}_{2} \mathrm{O}$ at $T=300 \mathrm{~K}$, superimposed with the profile matching plot (black line), the difference (blue line), and the Bragg positions (green ticks). The agreement Bragg Rfactor and $\chi^{2}$ are equal to 0.255 and 2.08 , respectively. (b) Thermal variation of the measured integrated intensity of the (001) diffraction peak observed for $2 \theta=8.6^{\circ}$ for $\left(\mathrm{NH}_{4}\right)_{5}\left[\mathrm{Mn}_{2} \mathrm{Cr}_{3}(\mathrm{ox})_{9}\right] \cdot 10 \mathrm{H}_{2} \mathrm{O}$. 


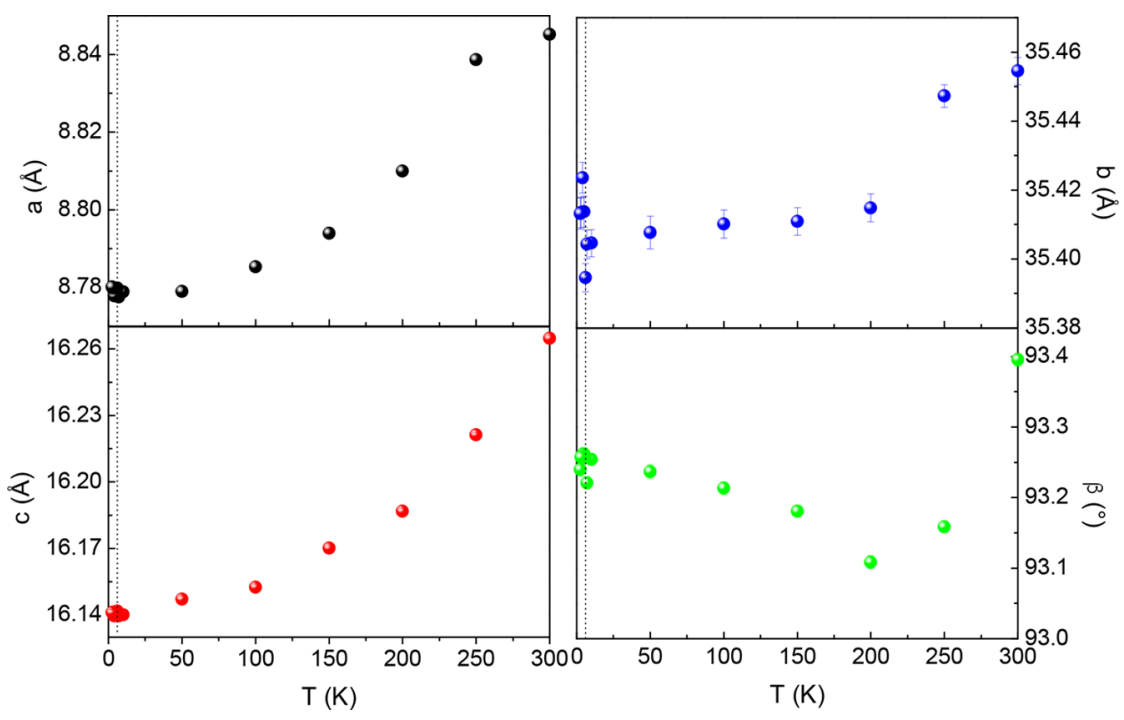

Figure 5. Thermal evolution of the structural parameters (unit cell lattice lengths and monoclinic angle) of $\left(\mathrm{NH}_{4}\right)_{5}\left[\mathrm{Mn}_{2} \mathrm{Cr}_{3}\left(\mathrm{ox}_{9}\right)_{9} \cdot 10 \mathrm{H}_{2} \mathrm{O}\right.$ extracted from profile matching of the neutron diffractograms. The vertical dotted lines show $T_{\mathrm{N}}$. When not visible, the standard error bars are smaller than the size of the symbols.

structural contribution. To isolate the magnetic contribution from the neutron diffraction pattern, the difference between the experimental patterns at $2.5 \mathrm{~K}$, below $T_{\mathrm{N}}$, and $7 \mathrm{~K}$, above $T_{\mathrm{N}}$, was calculated (Figure 6). Strikingly, only one peak is

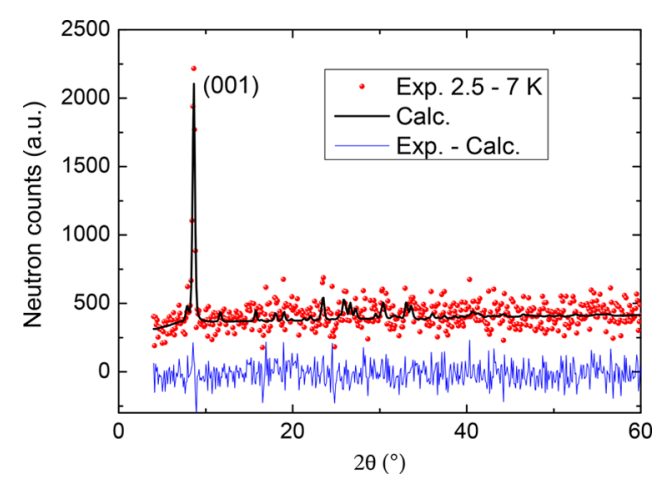

Figure 6. Difference (red circles) between the diffraction patterns for $\left(\mathrm{NH}_{4}\right)_{5}\left[\mathrm{Mn}_{2} \mathrm{Cr}_{3}(\mathrm{ox})_{9}\right] \cdot 10 \mathrm{H}_{2} \mathrm{O}$ measured at 2.5 and $7 \mathrm{~K}$, superimposed with the calculated magnetic model corresponding to the irreducible representation $\Gamma_{4}$ (black continuous line) as described in the text. The blue line is the difference between the measured and calculated magnetic diffractograms. The magnetic $\mathrm{R}$-factor and $\chi^{2}$ are equal to 46.6 and 19.3 , respectively. These high values reflect the low statistics.

clearly visible in the magnetic diffractogram. It can be indexed in the same space group as the nuclear contribution in agreement with a propagation vector $K=0$. Based on a careful use of group theory, this unique peak allows to get useful information about the magnetic structure. There are four irreducible representations, $\Gamma_{1}$ to $\Gamma_{4}$, in the group of the wave vector (equal to the $C 2 / c$ space group). For a second-order transition, a magnetic structure corresponding to one of these representations should be stabilized, the same for all coupled $\mathrm{Mn}$ (II) and $\mathrm{Cr}$ (III) magnetic moments. For each representation, the Fourier components corresponding to the $8 \mathrm{f}$ Wyckoff position associated with one $\mathrm{Cr}$ (III) site and to the $4 \mathrm{e}$ Wyckoff position associated with one $\mathrm{Cr}$ (III) site and two $\mathrm{Mn}$ (II) sites are reported in Table 1.

A Rietveld refinement of the magnetic diffractogram based on each of the four magnetic models was attempted by varying the components of the magnetic moments in agreement with the symmetry constraints. Nonetheless, due to the lack of structural refinement necessary to determine a global scaling factor, the determination of the absolute values of the magnetic moments was not possible. We therefore fixed the amplitude of the magnetic moments of the $\mathrm{Mn}$ (II) to $5 \mu_{\mathrm{B}}$ since the orbital angular momentum is expected to be close to zero. The magnetic moments of $\mathrm{Cr}$ (III) were also constrained to be equal for both sites. Representation $\Gamma_{4}$ was the only one yielding one dominant peak with a refined magnetic moments of $2.9 \pm 0.5 \mu_{\mathrm{B}}$ for $\mathrm{Cr}(\mathrm{III})$, compatible with the measured saturated magnetization at $2.5 \mathrm{~K}$ (Figure 6 and Table 2 for the magnetic moment components). For this calculation, we also constrained the $\mathrm{Cr}$ magnetic moments on the $8 \mathrm{f}$ site to lie in

Table 1. Fourier Components of the Four Irreducible Representations for both Wyckoff Sites $8 \mathrm{f}$ ( $1 \mathrm{Cr}$ Site) and 4e (1 Cr Site and $2 \mathrm{Mn}$ Sites) Corresponding to the Magnetic Structure of $\left(\mathrm{NH}_{4}\right)_{5}\left[\mathrm{Mn}_{2} \mathrm{Cr}_{3}(\mathrm{ox})_{9}\right] \cdot 10 \mathrm{H}_{2} \mathrm{O}$

\begin{tabular}{lllll}
\multicolumn{1}{c}{ site } & \multicolumn{1}{c}{$\Gamma_{1}$} & \multicolumn{1}{c}{$\Gamma_{2}$} & $\Gamma_{3}$ & $\Gamma_{4}$ \\
8f $(x, y, z)$ & $(u, v, w)$ & $(u, v, w)$ & $(u, v, w)$ & $(u, v, w)$ \\
8f $(-x, y,-z+1 / 2)$ & $(-u, v,-w)$ & $(-u, v,-w)$ & $(u,-v, w)$ & $(u,-v, w)$ \\
8f $(-x,-y,-z)$ & $(u, v, w)$ & $(-u,-v,-w)$ & $(u, v, w)$ & $(-u,-v,-w)$ \\
8f $(x,-y, z+1 / 2)$ & $(-u, v,-w)$ & $(u,-v, w)$ & $(u,-v, w)$ & $(-u, v,-w)$ \\
4e $(0, y, 1 / 4)$ & $(0, u, 0)$ & $(0, u, 0)$ & $(u, 0, v)$ & $(u, 0, v)$ \\
4e $(0,-y, 3 / 4)$ & $(0, u, 0)$ & $(0,-u, 0)$ & $(u, 0, v)$ & $(-u, 0,-v)$
\end{tabular}


Table 2. Refined Components of the Magnetic Moments for the Four Different Sites in the $\Gamma_{4}$ Irreducible

\section{Representation}

\begin{tabular}{lccc}
\multicolumn{1}{c}{ site } & $m_{x}(x)$ & $m_{y}(y)$ & \multicolumn{1}{c}{$m_{z}(z)$} \\
$\mathrm{Cr}(8 \mathrm{f})$ & $-2.4 \pm 0.5(0.3164)$ & $0(0.0728)$ & $-1.7 \pm 0.6(0.4353)$ \\
$\mathrm{Cr}(4 \mathrm{e})$ & $-2.6 \pm 0.5(0.0000)$ & $0(0.2911)$ & $1.0 \pm 0.9(0.2500)$ \\
$\mathrm{Mn}(4 \mathrm{e})$ & $-4.9 \pm 0.2(0.0000)$ & $0(0.4495)$ & $0.7 \pm 1.0(0.2500)$ \\
$\mathrm{Mn}(4 \mathrm{e})$ & $-4.1 \pm 0.6(0.0000)$ & $0(0.6946)$ & $2.7 \pm 0.9(0.2500)$ \\
\hline
\end{tabular}

the ac plane since it did not improve the fit to allow them to acquire a component along the $b$ axis. Note that imposing a zero $c$ component of the magnetic moments yields an unrealistically small value of the $\mathrm{Cr}$ magnetic moment (1.7 $\left.\mu_{\mathrm{B}}\right)$. The calculation of the whole magnetic diffractogram shows that other magnetic peaks are expected with much lower intensities that are, in the present case, below the noise level. Attempts to measure PND on deuterated samples did not improve this situation. Although certainly not univocal due to the large noise in the data preventing to measure the small peaks beyond the (001) one in the magnetic diffractogram (Figure 6), the main features of the magnetic structure seem quite robust, in particular, concerning the sign of the magnetic couplings.

The refinement of the magnetic neutron data corresponds to a complex organization of the magnetic moments of the $\mathrm{Mn}$ (II) and $\mathrm{Cr}$ (III) ions (Figure 7). The $\mathrm{Cr}$ and $\mathrm{Mn}$ magnetic moments have a main component along the $a$ axis and a smaller one along the $c$ axis, as shown in (a), leading to an antiferromagnetic structure with noncollinear magnetic moments. In this model, there are three different angles between the ferromagnetically coupled $\mathrm{Cr}$ and $\mathrm{Mn}$ magnetic moments of 11,44 , and $69^{\circ}$, respectively. The magnetic structure is then made of ferromagnetic sheets in the $(a b)$ planes (Figure $7 \mathrm{~b}$ ), antiferromagnetically coupled along the $c$ axis (Figure 7c).

\section{DISCUSSION}

While comparing Figures 1 and 7, an univocal relation between the bridging modes (bis(bidentate) vs monodentate-bidentate) and the nature of the isotropic exchange interaction (ferromagnetic vs antiferromagnetic) is clearly evidenced for $\left(\mathrm{NH}_{4}\right)_{5}\left[\mathrm{Mn}_{2} \mathrm{Cr}_{3}(\mathrm{ox})_{9}\right] \cdot 10 \mathrm{H}_{2} \mathrm{O}$. The magnetic structure refinement nicely confirms the robustness of the magnetostructural analysis. ${ }^{6}$ Note also that the magnetic structure corresponding to the $\Gamma_{4}$ irreducible representation does not allow any ferromagnetism, where all components of the magnetic moments for a given metal ion are compensated. On the other hand, it is compatible with a noncollinear arrangement of the magnetic moments on the different sites, especially those borne by the two metallic ions, both for the F and AF isotropic exchange interaction observed in the $(a b)$ planes and along the $c$ axis respectively. Given the rather low ZFS expected for chromium(III) and manganese(II) ions, this canting might rather originate from anisotropic interactions such as antisymmetric exchange interaction, which can occur locally through the moderate (bis(bidentate) mode) to strong (monodentate-bidentate mode) asymmetry of the oxalates bridges, despite the overall centrosymmetric character of the compound space group.

The present study shows the complementarity of neutron diffraction with more widespread magnetometry studies. It brings a fine comprehension of the intrinsically complex magnetic structures of molecule-based magnets related to their low global and local symmetries. ${ }^{18-22}$ Nonetheless, it has not been extensively used for the study of these compounds for essentially two reasons: (i) the need of nearly centimeter-scale single crystals or (ii) for studies on powder, the belief that fully deuterated samples together with the corresponding synthetic difficulties are unavoidable to obtain valuable results. As exemplified here, the improvement of the neutron sources and detectors has partly lifted these drawbacks.

\section{CONCLUDING REMARKS}

The long-range magnetic ordering of an atypical centrosymmetric 2D oxalate-based magnet $\left(\mathrm{NH}_{4}\right)_{5}\left[\mathrm{Mn}_{2} \mathrm{Cr}_{3}(\mathrm{ox})_{9}\right]$. $10 \mathrm{H}_{2} \mathrm{O}$ has been thoroughly studied by combining macroscopic magnetization measurements and microscopic neutron diffraction studies. This complementary approach has proven its efficiency. It indeed led to a successful determination, constrained by symmetry considerations, of the magnetic order of this coordination compound and a direct probe of the nature of its magnetic interactions. This analysis allows to take a further step forward and to predict other symmetry-allowed properties. For instance, the magnetic point group is $2^{\prime} / \mathrm{m}$ (magnetic space group $P 2^{\prime} / c$ ) resulting from the loss of the

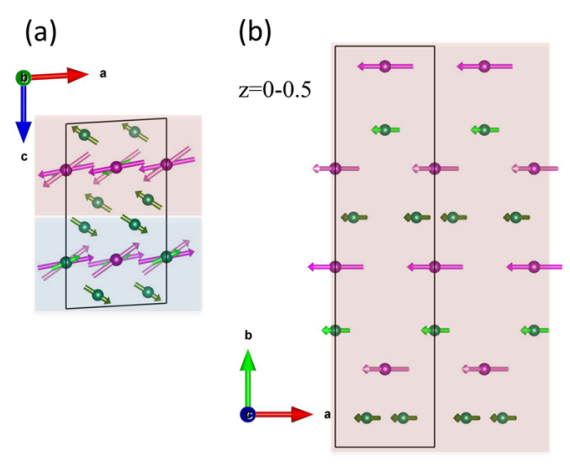

(c)

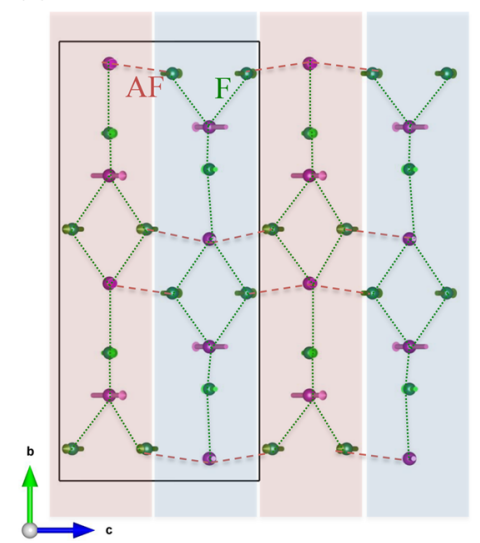

Figure 7. Magnetic structure of $\left(\mathrm{NH}_{4}\right)_{5}\left[\mathrm{Mn}_{2} \mathrm{Cr}_{3}(\mathrm{ox})_{9}\right] \cdot 10 \mathrm{H}_{2} \mathrm{O}$ deduced from PND. (a) Projection in the (ac) plane. (b) Top: projection in the $(a b)$ plane with a ferromagnetic slice for $c$ within $[0,0.5]$. (c) Slice in the $(b c)$ plane showing the ferromagnetic (green) and antiferromagnetic (red) exchange interactions. The colored boxes represent the antiferromagnetic stacking of ferromagnetic sheets. 
inversion center combined with time reversal in the magnetic arrangement. This authorizes a linear magnetoelectric tensor, which has a nondiagonal form with nonequal off-diagonal terms as in $\mathrm{MnPS}_{3}{ }^{23}$ It means that an electric polarization is allowed by symmetry to be induced perpendicular to the magnetic field generating it and vice versa for a ferromagnetic contribution induced by an electric field. This special form of the magnetoelectric tensor is associated with ferrotoroidicity ${ }^{24}$ establishing $\left(\mathrm{NH}_{4}\right)_{5}\left[\mathrm{Mn}_{2} \mathrm{Cr}_{3}(\mathrm{ox})_{9}\right] \cdot 10 \mathrm{H}_{2} \mathrm{O}\left(\mathrm{ox}^{2-}=\mathrm{C}_{2} \mathrm{O}_{4}{ }^{2-}\right)$ as a potential multifuntionnal molecular magnet.

\section{AUTHOR INFORMATION}

\section{Corresponding Author}

*E-mail: cyrille.train@lncmi.cnrs.fr.

\section{ORCID}

Sylvie Ferlay: 0000-0002-9707-9514

Cyrille Train: 0000-0002-6726-2086

\section{Present Addresses}

${ }^{\perp}$ Institute of Mechanics, Materials and Civil Engineering, Université catholique de Louvain, Louvain-la-Neuve, Belgium. \#University of Bucharest, Faculty of Chemistry, Inorganic Chemistry Laboratory, Str. Dumbrava Rosie nr. 23, 020464 Bucharest, Romania.

\section{Author Contributions}

The manuscript was written through contributions of all authors. All authors have given approval to the final version of the manuscript.

\section{Notes}

The authors declare no competing financial interest.

\section{ACKNOWLEDGMENTS}

The authors are grateful to E. Suard and C. Ritter for their help in the attempts to measure the samples by neutron diffraction at Institut Laue-Langevin. The French National Research Agency (ANR) is acknowledged for financial support through Monafer (ANR-18-CE09-0032) projects.

\section{REFERENCES}

(1) Clément, R.; Decurtins, S.; Gruselle, M.; Train, C. Polyfunctional Two- (2D) and Three- (3D) Dimensional Oxalate Bridged Bimetallic-based Magnets. Monatsh. Chem. 2003, 134, 117-135.

(2) Gruselle, M.; Train, C.; Boubekeur, K.; Gredin, P.; Ovanesyan, N. Oxalate-based Optically Active Multifunctional Magnets. Coord. Chem. Rev. 2006, 250, 2491-2500.

(3) Sadakiyo, M.; Yamada, T.; Kitagawa, H. Rational Designs for Highly Proton-Conductive Metal-Organic Frameworks. J. Am. Chem. Soc. 2009, 131, 9906-9907.

(4) Pardo, E.; Train, C.; Gontard, G.; Boubekeur, K.; Fabelo, O.; Liu, H.; Dkhil, B.; Lloret, F.; Nakagawa, K.; Tokoro, H.; Ohkoshi, S.I.; Verdaguer, M. High Proton Conduction in a Chiral Ferromagnetic Metal-Organic Quartz-like Framework. J. Am. Chem. Soc. 2011, 133, 15328-15331.

(5) Pardo, E.; Train, C.; Boubekeur, K.; Gontard, G.; Cano, J.; Lloret, F.; Nakatani, K.; Verdaguer, M. Topological Versatility of Oxalate-Based Bimetallic One-Dimensional (1D) Compounds with Ammonium Cations. Inorg. Chem. 2012, 51, 11582-11593.

(6) Maxim, C.; Ferlay, S.; Tokoro, H.; Ohkoshi, S.-I.; Train, C. Atypical Stoichiometry for a 3D Bimetallic Oxalate-Based Long-range Ordered Magnet Exhibiting High Proton Conductivity. Chem. Commun. 2014, 50, 5629-5632.

(7) Maxim, C.; Ferlay, S.; Train, C. Dialkyl substituted monoamidinium-templated oxalate-based non centro-symmetric 2D compounds. C. R. Chim. 2019, 22, 534-540.
(8) Tamaki, H.; Zhong, Z. J.; Matsumoto, N.; Kida, S.; Koikawa, M.; Achiwa, N.; Hashimoto, Y.; Okawa, H. Design of metal-complex magnets. Syntheses and magnetic properties of mixed-metal assemblies $\left\{\mathrm{NBu}_{4}\left[\mathrm{MCr}\left(\mathrm{C}_{2} \mathrm{O}_{4}\right)_{3}\right]\right\}_{\mathrm{x}}\left(\mathrm{NBu}_{4}{ }^{+}=\right.$tetra(n-butyl)ammonium ion; ox ${ }^{2-}=$ oxalate ion; $\mathrm{M}=\mathrm{Mn}^{2+}, \mathrm{Fe}^{2+}, \mathrm{Co}^{2+}, \mathrm{Ni}^{2+}$, $\left.\mathrm{Cu}^{2+}, \mathrm{Zn}^{2+}\right)$. J. Am. Chem. Soc. 1992, 114, 6974-6979.

(9) Decurtins, S.; Schmalle, H. W.; Schneuwly, P.; Ensling, J.; Guetlich, P. A Concept for the Synthesis of 3-Dimensional Homoand Bimetallic Oxalate-Bridged Networks $\left[\mathrm{M}_{2}(\mathrm{ox})_{3}\right]_{\mathrm{n}}$. Structural, Moessbauer, and Magnetic Studies in the Field of Molecular-Based Magnets. J. Am. Chem. Soc. 1994, 116, 9521-9528.

(10) Maxim, C.; Ferlay, S.; Train, C. Binuclear heterometallic $\mathrm{M}(\mathrm{III})-\mathrm{Mn}(\mathrm{II})(\mathrm{M}=\mathrm{Fe}, \mathrm{Cr})$ oxalate-bridged complexes associated with a bisamidinium dication: a structural and magnetic study. New J. Chem. 2011, 35, 1254-1259.

(11) Maxim, C.; Pardo, E.; Hosseini, M. W.; Ferlay, S.; Train, C. The odd association of a $\mathrm{C} 3 \mathrm{~h}$ trisamidinium cation and tosylate anion with a series of linear oxalate-bridged trinuclear heterometallic complexes. Dalton Trans. 2013, 42, 4704-4713.

(12) Coronado, E.; Galan-Mascaros, J. R.; Mart1-Gastaldo, C.; Martınez, A. M. Heptacoordinated $\mathrm{Mn}^{\mathrm{II}}$ in oxalate-based bimetallic 2D magnets: synthesis and characterisation of $\left[\mathrm{Mn}(\mathrm{L})_{6}\right][\mathrm{Mn}$ $\left.\left(\mathrm{CH}_{3} \mathrm{OH}\right) \mathrm{M}^{\mathrm{III}}(\mathrm{ox})_{3}\right]_{2}\left(\mathrm{M}^{\mathrm{III}}=\mathrm{Cr}, \mathrm{Rh}\right.$; ox = oxalate dianion; $\mathrm{L}=$ $\left.\mathrm{H}_{2} \mathrm{O}, \mathrm{CH}_{3} \mathrm{OH}\right)$. Dalton Trans. 2006, 3294-3299.

(13) Pardo, E.; Train, C.; Liu, H.; Chamoreau, L. M.; Dkhil, B.; Boubekeur, K.; Lloret, F.; Nakatani, H.; Tokoro, H.; Ohkoshi, S.-I.; Verdaguer, M. Multiferroics by rational design: implementing ferroelectricity in molecule-based magnets. Angew. Chem., Int. Ed. 2012, 51, 8356-8360.

(14) Serezhkin, V. N.; Artem'eva, M. Y.; Serezhkina, L. B.; Mikhailov, Y. N. Crystal-Chemical Role of Oxalate Ions. Russ. J. Inorg. Chem. 2005, 50, 1019-1030.

(15) Pascal, P. Magnetochemical studies. Ann. Chim. Phys. 1910, 19, 5-70.

(16) Rodrigues-Carvajal, J. Software FULLPROF-98, Version 5.06, 2015.

(17) Andrès, R.; Gruselle, M.; Malézieux, B.; Verdaguer, M.; Vaissermann, J. Enantioselective Synthesis of Optically Active Polymeric Homo- and Bimetallic Oxalate-Bridged Networks $\left[\mathrm{M}_{2}(\mathrm{ox})_{3}\right]_{\mathrm{n}}$. Inorg. Chem. 1999, 38, 4637-4646.

(18) Ressouche, E. Investigating molecular magnetism with polarized neutrons. Phys. B 1999, 267-268, 27-36.

(19) Gillon, B.; Mathonière, C.; Ruiz, E.; Alvarez, S.; Cousson, A.; Rajendiran, T. K.; Kahn, O. Spin Densities in a Ferromagnetic Bimetallic Chain Compound: Polarized Neutron Diffraction and DFT Calculations. J. Am. Chem. Soc. 2002, 124, 14433-14441.

(20) Gillon, B.; Goujon, A.; Willemin, S.; Larionova, J.; Desplanches, C.; Ruiz, E.; André, G.; Stride, J. A.; Guérin, C. Neutron Diffraction and Theoretical DFT Studies of Two Dimensional Molecular-Based Magnet $\mathrm{K}_{2}\left[\mathrm{Mn}\left(\mathrm{H}_{2} \mathrm{O}\right)_{2}\right]_{3}\left[\mathrm{Mo}(\mathrm{CN})_{7}\right]_{2} \cdot 6 \mathrm{H}_{2} \mathrm{O}$. Inorg. Chem. 2007, 46, 1090-1099.

(21) Nuttall, C. J.; Day, P. The Magnetic Structures of the Layer Ferri- magnets $\mathrm{P}\left(\mathrm{C}_{6} \mathrm{D}_{5}\right)_{4} \mathrm{M}^{\mathrm{II}} \mathrm{Fe}\left(\mathrm{C}_{2} \mathrm{O}_{4}\right)_{3}\left(\mathrm{M}^{\mathrm{II}}=\mathrm{Mn}, \mathrm{Fe}\right)$. Inorg. Chem. 1998, 37, 3885-3888.

(22) Pointillart, F.; Gruselle, M.; André, G.; Train, C. Are optically active three-dimensional oxalate-based ferromagnets good candidates for the observation of X-rays Magnetochiral dichroism? A neutron diffraction study. J. Phys.: Condens. Matter 2008, 20, 135214.

(23) Ressouche, E.; Loire, M.; Simonet, V.; Ballou, R.; Stunault, A.; Wildes, A. Magnetoelectric MnPS3 as a candidate for ferrotoroidicity. Phys. Rev. B 2010, 82, 100408.

(24) Schmid, H. Some symmetry aspects of ferroics and single phase multiferroics. J. Phys.: Condens. Matter 2008, 20, 434201. 